\title{
PERITONITIS MECONIAL PSEUDOQUÍSTICA POR ATRESIA INTESTINAL PERFORADA. REPORTE DE UN CASO
}

\section{PSEUDOCHISTIC MECONIAL PERITONITIS DUE TO PERFORATED INTESTINAL ATRESIA. A CASE REPORT}

\begin{abstract}
Carlos Cabrera Valerio ${ }^{a}$, Niurka González ${ }^{1 \mathrm{~b}}$, Andreina Moreno Reyes ${ }^{\mathrm{c}}$, Ariadna García Risk ${ }^{\mathrm{c}}$, Noelia Madera ${ }^{\mathrm{c}}$. RESUMEN

La peritonitis meconial secundaria a perforación intestinal es una complicación temida de la atresia intestinal, puesto que incrementa la morbimortalidad perinatal. Aunque sigue siendo una presentación rara, su diagnóstico debe de hacerse temprano para evitar complicaciones. Presentamos un caso clínico de un recién nacido de 38 semanas sin antecedentes prenatales indicativos de obstrucción intestinal. Quien presenta vómitos biliosos/ fecaloideos, no meconización a las 12 horas de vida extrauterina, por lo cual se le realiza radiografía de abdomen de pie y acostada que muestra marcada distensión de asas intestinales, ausencia de aire distal, edema interasas. Se realiza laparotomía exploratoria y se hace yeyunostomía para luego re-intervenirla (second look), encontrando mejoría de asas intestinales, logrando una anastomosis yeyuno-ileal término-terminal. La operación temprana se puede considerar un factor de importancia para mejorar el resultado de los pacientes con peritonitis por meconio, de igual forma se necesita de un diagnóstico temprano.
\end{abstract}

Palabras Clave: Atresia Intestinal; Quiste Meconial; Peritonitis Meconial; Perforación intestinal; Recién Nacido (Fuente: DeCS BIREME).

\begin{abstract}
Meconium peritonitis secondary to intestinal perforation is a feared complication of intestinal atresia, since it increases perinatal morbidity and mortality. Although it is still a rare presentation, its diagnosis must be made early to avoid complications. This case presents a clinical case of a 38-week-old newborn with no prenatal history indicative of intestinal obstruction who presents bilious / fecaloid vomiting, no meconization at 12 hours of extrauterine life, for which a standing and lying abdominal $\mathrm{X}$-ray is performed showing marked distension of the intestinal loops, absence of distal air, interloop edema. An exploratory laparotomy and jejunostomy are performed to later re-intervene (second look), finding improvement in intestinal loops and achieving a jejunal-ileal end-to-end anastomosis. Early operation can be considered an important factor to improve the outcome of patients with meconium peritonitis, in the same way an early diagnosis is needed.
\end{abstract}

Key Words: Intestinal atresia; Meconium cyst; Meconium Peritonitis; Intestinal perforation; Newborn (Source MeSH NLM).

\section{INTRODUCCIÓN}

La peritonitis por meconio es una inflamación química estéril del peritoneo por perforación intestinal intrauterina. Esto ocurre en 1:30,000 nacimientos con una mortalidad reportada de más del $80 \%{ }^{1}$. La contaminación peritoneal con meconio antes de nacer o en el periodo neonatal inmediato produce peritonitis grave.

La peritonitis meconial secundaria a perforación intestinal es una complicación temida de la atresia ileal puesto que incrementa la morbimortalidad perinatal. El $10 \%$ de los lactantes con atresia intestinal padece peritonitis por meconio ${ }^{2}$. Aunque sigue siendo una presentación rara, su diagnóstico debe de hacerse temprano para evitar complicaciones y reducir su morbimortalidad ${ }^{3}$.
La atresia intestinal perforada con peritonitis meconial es una presentación menos usual que la atresia intestinal aislada. A continuación se presenta caso clínico en recién nacido de 38 semanas sin antecedentes prenatales indicativos de obstrucción intestinal.

\section{REPORTE DE CASO}

Recién nacido de sexo femenino de 24 horas de vida extrauterina, hija de madre de 26 años de edad, $\mathrm{G} 1, \mathrm{P0}, \mathrm{A0}$ con antecedente mórbido de anemia durante el embarazo manejada con hierro y ácido fólico, con término del embarazo a las 38 semanas, vía cesárea; obteniendo producto único, con pobre llanto al nacer, distensión abdominal marcada con red venosa visible, y

\footnotetext{
Servicio de Cirugía Pediátrica del Departamento Materno Infantil del Hospital Provincial Francisco A. Gonzalvo. La Romana, República Dominicana.

a Cirujano Pediatra, Profesor de Pediatría de la Universidad Pedro Henríquez Ureña -UNPHU- Medico ayudante del servicio de Cirugía Pediátrica del Hospital Infantil Dr. Robert Reíd Cabral, Miembro titular de la Asociación Dominicana de Investigadores Pediátricos

Cirujana Pediatra, Servicio de Cirugía Pediátrica del Hospital Provincial Francisco A. Gonzalvo. La Romana, República Dominicana

Médico General, Universidad Iberoamericana -UNIBE

(iD Código ORCID: https://orcid.org/0000-0003-2576-3912: Carlos Cabrera Valerio

Citar como: Cabrera Valerio C, Gonzáles N, Moreno Reyes A, García Risk A, Madera N. Peritonitis meconial pseudoquística por atresia intestinal perforada. Reporte de un caso. Rev Peru Investig Matern Perinat. 2021;10(1): 37-40 DOI https://doi.org/10.33421/inmp.2021220
}

Recibido: 13-02-2021 Aprobado: 31-03-2021 
a las 12 horas presenta vómitos biliosos/ fecaloideos, no meconización, se le realiza radiografía de abdomen de pie y acostada que muestra distensión de asas intestinales, ausencia de aire distal, edema interasas, (ver Figura 1). Se le realiza laparotomía exploratoria por incisión transversa derecha supraumbilical, encontrando quiste meconial con 4 perforaciones intestinales: 3 perforaciones en íleon, siendo necesaria la resección y anastomosis íleo ileal más yeyunostomía para realizar un second look, debido a que quiste meconial envolvía íleon distal y colon con intestino desvitalizado y friable. Al quinto día paciente se le realiza second look, encontrando mejoría de asas intestinales, se reseca membrana del quiste restante en cavidad, lisis de bridas y adherencias, lavado de cavidad peritoneal, anastomosis yeyuno ileal término terminal logrando finalmente la recanalización intestinal (ver Figura 2), quedando con aproximadamente $50 \mathrm{~cm}$ de longitud de intestino delgado con su válvula ileocecal. Paciente se manejó en UCIN, sin necesidad de ventilación mecánica, con oxigenoterapia por cánula binasal, con antibioterapia a base de Meropenem, Vancomicina, Fluconazol, Paracetamol infusión como analgesia y alimentación parenteral parcial vía catéter venoso central yugular interno derecho.

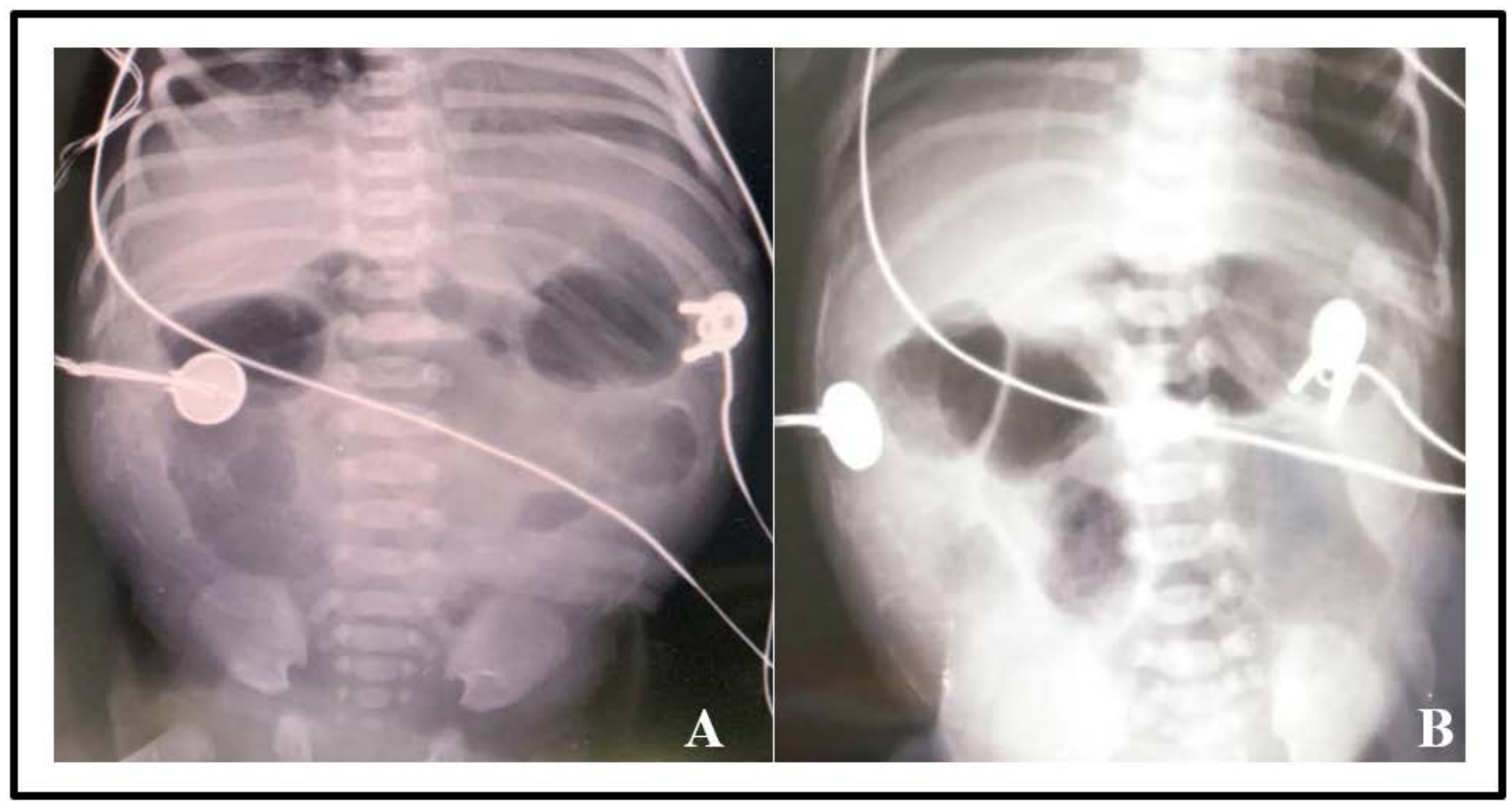

Figura 1: Radiografía de abdomen simple a las 12 horas de recién nacida: A- Proyección AP de pie donde se observa asas delgada dilatadas, con ausencia de aire distal. B- Proyección AP acostada con asas intestinales dilatadas, edema interasa.
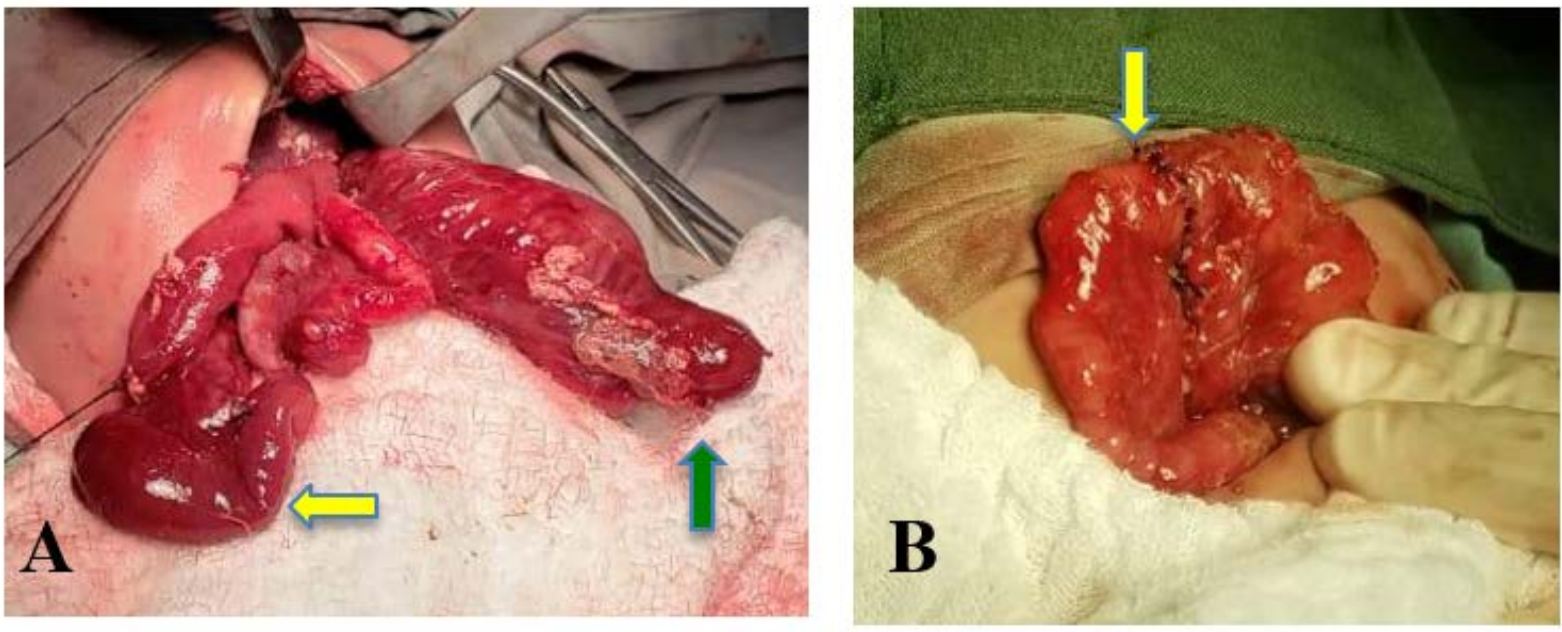

Figura 2: A- Los segmentos de intestino delgado viables, luego de la resección del tejido desvitalizado y membrana seudoquística flecha verde intestino proximal (yeyuno), flecha amarilla intestino distal (íleon). B- Flecha amarilla indica la anastomosis terminoterminal yeyunoileal. 


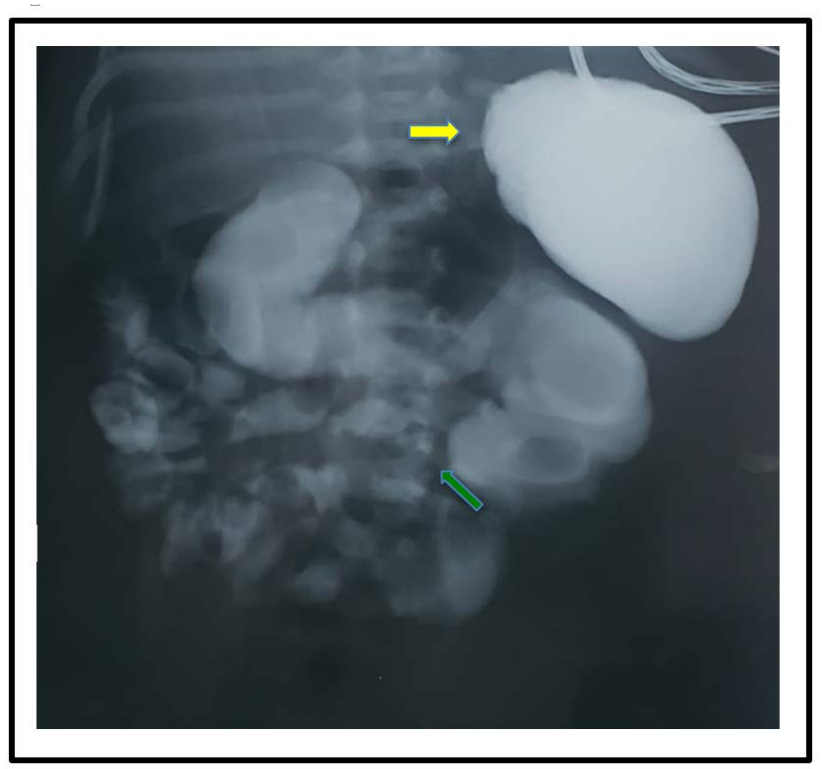

Figura 3: Radiografía con medio de contraste hidrosoluble, donde la flecha amarilla indica el estómago, flecha verde lugar donde se observa zona de transición de intestino dilatado a intestino más delgado, correspondiente al lugar de anastomosis.

Paciente con evolución satisfactoria, presentando 2-3 vómitos biliosos luego de retirar la sonda nasogástrica después de un lapso de 5 días, se realiza tránsito intestinal donde no se visualiza fuga de la anastomosis (Ver Figura 3 ), manejándose con procinético oral, finalmente logrando una tolerancia adecuada de leche materna y se egresa a los 12 días de su post-quirúrgico.

\section{DISCUSIÓN}

Peritonitis por meconio (PM), una peritonitis química estéril causada por perforación del intestino intrauterino, es una condición rara, con una incidencia estimada de 1 de cada 30,000 nacimientos. Causas subyacentes de las perforaciones son: atresia del intestino delgado generalmente causada por accidentes de oclusión vascular, íleo meconial, hernia interna, invaginación intestinal, vólvulo, enfermedad de Hirschsprung y rotura apendicular intrauterina ${ }^{1,4-5}$. Existe una asociación entre peritonitis meconial y fibrosis quística, sin embargo, no se realizó un tamizaje neonatal ni prueba de sudor en nuestro paciente por límite de recursos económicos, de todos modos hubo una evolución favorable luego de la anastomosis por lo que no se sospechó posteriormente. La presentación clínica de la PM en el recién nacido se clasifica en cuatro tipos: fibroadhesiva, pseudoquiste, ascitis e infecciosa ${ }^{6}$. Nuestro caso corresponde a pseudoquiste meconial.

La atresia intestinal es la causa más frecuente de obstrucción intestinal en el recién nacido, con una incidencia de uno por cada 1000 nacimientos y menos del $10 \%$ de los lactantes con atresia intestinal padece peritonitis por meconio ${ }^{3,5}$. En un entorno de bajos recursos donde se carece de atención quirúrgica tiene alta mortalidad y morbilidad. Chan KW et al asegura que con la mejora en los cuidados intensivos neonatales, la intervención quirúrgica oportuna y la nutrición del lactante, la tasa de mortalidad se redujo de más del $90 \%$ al $11 \%{ }^{6,7}$.

La presencia de polihidramnios materno y asas intestinales dilatadas son características sugestivas de atresia yeyunoileal prenatal ${ }^{6}$. La ecografía prenatal donde se encuentra la presencia de calcificaciones y asas intestinales dilatadas, sugiere fuertemente la posibilidad de $\mathrm{PM}^{7,8}$. Nuestro paciente tenía varias ecografías obstétricas, pero sin reporte de los hallazgos descrito, las desventaja de este método diagnóstico radica en que es operador dependiente. Es en la ecografía prenatal donde se encuentra la presencia de calcificaciones y asas intestinales dilatadas, sugiere fuertemente la posibilidad de $\mathrm{PM}^{4}$.

La radiografía simple abdominal es indispensable para realizar un diagnóstico precoz en un paciente con sospecha de obstrucción intestinal. En nuestro caso se evidenció marcada distensión de asas intestinales, ausencia de aire distal y edema interasas.

La PM es una patología grave del período neonatal cuya morbimortalidad asociada puede ser atenuada mediante una adecuada sospecha diagnóstica y un manejo expectante en el período neonatal, para definir la mejor alternativa terapéutica, que va desde la evolución espontánea hasta exploración quirúrgica abdominal con resección intestinal y ostomía o anastomosis primaria, siendo la recanalización primaria la de mayor ventaja ya que disminuye la estancia hospitalaria, evita la morbilidad relacionada con el estoma y la segunda laparotomía para el cierre del estoma ${ }^{5,6}$.

En un estudio retrospectivo realizado en Hong Kong establece que se puede realizar una anastomosis primaria en el $94 \%$ de los pacientes con atresia yeyunoileal asociada a peritonitis meconial pseudoquística, donde las tasas de mortalidad y reintervención fueron más bajas ${ }^{6}$. Existen diferentes criterios para el manejo adecuado de la peritonitis meconial, sin embargo, el desenlace de estos pacientes dependerá los datos ecográficos prenatales, la presentación clínica del neonato, el diagnóstico pre o postnatal, la edad gestacional y el peso del neonato, los cuales indicarán al especialista el empleo de la mejor intervención.

En el caso de nuestra paciente, la radiografía de abdomen muestra una marcada distensión de las asas intestinales, ausencia de aire distal y edema interasas, junto con síntomas a las 12 horas de nacida, indica una alta sospecha obstrucción intestinal. De acuerdo a estos hallazgos, se realiza una primera intervención donde se encuentra una gran membrana con contenido meconial, 
con segmentos desvitalizados. Riley et al, notificaron un caso con PM donde se colocó un drenaje abdominal en los primeros minutos de vida y se extrajo la ascitis biliosa, luego fue llevado al quirófano para exploración, resección intestinal y anastomosis primaria ${ }^{9}$. En nuestra paciente se realizó una intervención exploratoria evacuatoria de la peritonitis, con lavado terapéutico de la cavidad en las primeras 24 horas de vida, lo que disminuyó el riesgo de sepsis y mortalidad, además se decide ostomizar para en una segunda intervención realizar resección intestinal no viable, se delimitaron bien los segmentos atrésicos, con una anastomosis termino-terminal de forma diferida.

Dado que, la operación temprana se puede considerar un factor de importancia para mejorar el resultado de los pacientes con peritonitis por meconio, de igual forma se necesita de un diagnóstico temprano. La resonancia magnética (RM) proporciona información adicional útil sobre la distribución del meconio en el intestino delgado, lo que ayuda a aclarar el nivel de obstrucción ${ }^{10,11}$. A pesar de los altos beneficios que puede brindar la RM en casos de Peritonitis meconial, en los países en vía de desarrollo (presente reporte de caso) su uso es limitado debido al alto costo del estudio. También debemos destacar que existe mucha debilidad en el diagnóstico prenatal por ecografías que carecen de criterios o hallazgos sospechosos de obstrucción intestinal.

En conclusión, la peritonitis por meconio está asociada con la atresia intestinal. La atención prenatal adecuada es necesaria para mejorar el pronóstico de los pacientes, ya que alerta la sospecha diagnóstica y se define la conducta a seguir en el periodo neonatal. Los pacientes con hallazgos de peritonitis meconial deben ser transferidos a hospitales terciarios. Se recomienda proceder con una anastomosis primaria, siempre verificar los daños tisulares y hallazgos quirúrgicos, en los casos necesarios una segunda intervención donde se realice una anastomosis diferida.

Financiamiento: Autofinanciado.

Declaración de conflictos de intereses: Los autores declaran no tener conflicto de intereses.

\section{REFERENCIAS BIBLIOGRÁFICAS}

1. Chandrasekaran N, Benardete D, Cariello L, et al. Prenatal sonographic diagnosis of meconium peritonitis from duodenal atresia. Case Reports 2017;2017:bcr2017219208. Disponible en: https://europepmc.org/article/pmc/ pmc5293971.

2. Tato Varela $\mathrm{S}$, et al. Diagnóstico prenatal de atresia ileal asociada a peritonitis meconial. Prog Obstet Ginecol. 2015. Disponible en: http://dx.doi.org/10.1016/j.pog.2015.07.011
3. Keith W. Ashcraft, MD. Cirugía Pediátrica, Atresia y estenosis intestinales. McGraw Hill; 2002: 429-441

4. Shinar S, Agrawal S, Ryu M, Van Mieghem T, Daneman A, Ryan G, Zani A, Chiu P, Chitayat D. Fetal Meconium Peritonitis - Prenatal Findings and Postnatal Outcome: A Case Series, Systematic Review, and Meta-Analysis. Ultraschall Med. 2020 Jun 23. English. doi: 10.1055/a1194-4363. Epub ahead of print. PMID: 32575129. Disponible en: https://pubmed.ncbi.nlm.nih.gov/32575129/

5. Saitua F, Lopetegui S, Soto F. Meconial peritonitis: A clinical experience. Rev. chil. pediatr. 2011 June. 82( 3 ): 218-224. Disponible en: https://scielo.conicyt.cl/scielo.php?script=sci_arttext\&pid=S0370-

6. Miyake H, Urushihara N, Fukumoto K, Sugiyama A, Fukuzawa $\mathrm{H}$, Watanabe K, Mitsunaga M, Kusafuka J, Hasegawa S. Primary anastomosis for meconium peritonitis: first choice of treatment. J Pediatr Surg. 2011 Dec;46(12):2327-31. Disponible en: https://www.jpedsurg.org/article/S00223468(11)00770-6/fulltext

7. Santana-Ortiz R, Zúñiga-Morán A, Aguirre-Jáuregui Ó, et al. Peritonitis meconial. ¿Anastomosis primaria o derivación y anastomosis diferida?. Rev Med MD. 2012;3.4(2):58-62.

8. Chan KW, Lee KH, Wong HY, Tsui SY, Wong YS, Pang KY, Mou JW, Tam YH. Cystic meconium peritonitis with jejunoileal atresia: Is it associated with unfavorable outcome? World J Clin Pediatr. 2017 Feb 8;6(1):40-44. Doi: 10.5409/ wjcp.v6.i1.40. PMID: 28224094; PMCID: PMC5296628. Disponible en: https://pubmed.ncbi.nlm.nih.gov/28224094/

9. Kitamura R, Midulla P, Mirensky T. Meconium Peritonitis Following Intestinal Atresia: A Case Report. Journal of Pediatric Surgery Case Reports. 2016 March. Disponible en: https://www.sciencedirect.com/science/article/ pii/S2213576616300021

10. Jiang Y, Pan W, Wu W, Wang W, Sun S, Wang J. Can early surgery improve the outcome of patients with meconium peritonitis? A single-center experience over 16years. BMC Pediatr. 2019 Dec 3;19(1):473. doi: 10.1186/s12887019-1844-5. PMID: 31795969; PMCID: PMC6889670. Disponible en: https://pubmed.ncbi.nlm.nih.gov/31795969/

11. Lu, Y., Ai, B., Zhang, W. et al. Fetal magnetic resonance imaging contributes to the diagnosis and treatment of meconium peritonitis. BMC Med Imaging 20, 55. 2020 May. Disponible en: $h$ ttps://doi.org/10.1186/s12880-020-00453-8

\section{Correspondencia:}

Carlos Manuel Cabrera Valerio

Dirección: Calle Bartolomé Colón No. 20, Villa Consuelo, Distrito Nacional, República Dominicana.

E-mail: carloscabrera84@gmail.com / info@drcabreravalerio.com Teléfono: +1-809-350-8652 